\title{
NW-G01, a novel cyclic hexapeptide antibiotic, produced by Streptomyces alboflavus 313: II. Structural elucidation
}

Zhengyan Guo, Zhiqin Ji, Jiwen Zhang, Jing Deng, Ling Shen, Wei Liu and Wenjun Wu

The Journal of Antibiotics (2010) 63, 733; doi:10.1038/ja.2010.116

Correction to: The Journal of Antibiotics (2010) 63, 231-235; doi:10.1038/ja.2010.24

The authors of the above article noted some errors in publication of the above paper (AOP and in May issue).

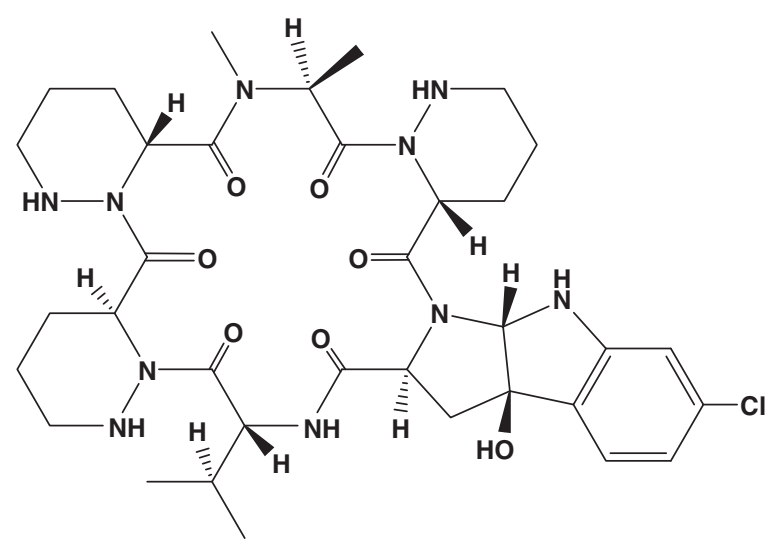

Figure 1 The structure of NW-G01.
1. On page 234 , right column, line 7 , regarding the wrong retention time of the FDAA derivatives of the acid hydrolysate of NW-G01. The corrected stereochemistry configuration of valine-derived amino acid is D-valine.

2. In Figures 1 and 3, regarding the wrong spatial configuration of a carbon atom (C34). The corrected figures and captions are shown below.

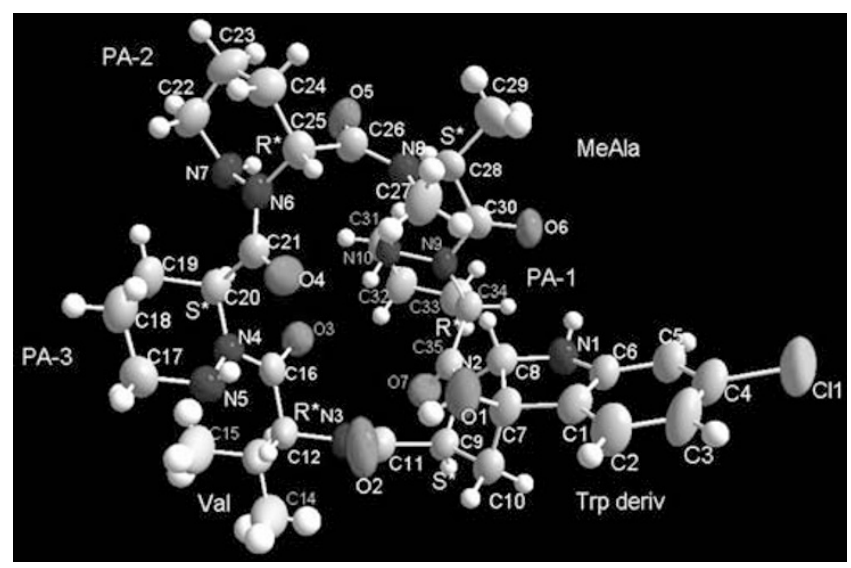

Figure 3 Molecular single-crystal X-ray diffraction photo of compound NW-G01. 